\title{
Mild behavioral impairment is related to frailty in non-dementia older adults: a cross-sectional study
}

\author{
Shaoyi Fan ${ }^{1}$, Ximin Liang ${ }^{1}$, Tianchan Yun ${ }^{1}$, Zhong Pei ${ }^{2}$, Bin $\mathrm{Hu}^{3}$, Zahinoor Ismail ${ }^{4}$, Zhimin Yang ${ }^{5^{*}}$ and Fuping $\mathrm{Xu}^{5^{*}}$
}

\begin{abstract}
Background: Frailty and cognitive decline are highly prevalent among older adults. However, the relationship between frailty and mild behavioral impairment (MBI), a dementia risk syndrome characterized by later-life emergence of persistent neuropsychiatric symptoms, has yet to be elucidated. We aimed to evaluate the associations between $\mathrm{MBI}$ and frailty in older adults without dementia.

Methods: In this cross-sectional study, a consecutive series of 137 older adults without dementia in the Anti-Aging Study, recruited from primary care clinics, were enrolled. Frailty was estimated using the Fried phenotype. MBI was evaluated by the Mild Behavioral Impairment Checklist (MBI-C) at a cut-off point of $>8$. Cognition was assessed with the Chinese versions of the Montreal Cognitive Assessment (MoCA-BC) and Mini-mental State Examination (MMSE). Multivariable logistic regression was performed to estimate the relationship between MBI and objective cognition with frailty status.

Results: At baseline, 30.7\% of the older adults had frailty and 18.2\% had MBI (MBI+ status). Multivariable logistic regression analysis demonstrated that compared to those without $\mathrm{MBI}$ (MBI- status), $\mathrm{MBI}+$ was more likely to have frailty (odds ratio $[\mathrm{OR}]=7.44,95 \% \mathrm{Cl}=1.49-37.21, p=0.02$ ). Frailty and $\mathrm{MBI}$ were both significantly associated with both MMSE and MoCA-BC score $(p<0.05)$.

Conclusions: Both frailty and MBI status were associated with higher odds of cognitive impairment. MBI was significantly associated with an increased risk of having frailty in the absence of dementia. This association merits further study to identify potential strategies for the early detection, prevention and therapeutic intervention of frailty.
\end{abstract}

Keywords: Cognitive impairment, Frailty, Mild behavioral impairment, Neuropsychiatric symptoms

\section{Background}

Frailty is a common geriatric condition presenting as a clinical state of decreased physiological reserve, increased vulnerability to death and increased susceptibility to even small stressors [1]. It is associated with an increased risk of adverse health-related outcomes,

\footnotetext{
*Correspondence: yangyo@vip.tom.com; xufuping163@163.com

${ }^{5}$ The Second Affiliated Hospital of Guangzhou University of Chinese Medicine, Guangdong Provincial Hospital of Chinese Medicine, 111 Dade Road, Guangzhou, People's Republic of China

Full list of author information is available at the end of the article
}

including falls, disability and mortality [2]. The prevalence of frailty is 3.9 to $51.4 \%$ among communitydwelling people aged 60 years and older, and the incidence increases with age [3]. As population aging has become a global phenomenon, frailty has become an emerging public health issue. To date, most definitions have prioritized the physical dimension of frailty, which includes symptoms and signs such as weight loss, muscle weakness, slower gait speed, and sedentary behavior [4]. Frailty has been most commonly operationalized using a

(c) The Author(s). 2020 Open Access This article is licensed under a Creative Commons Attribution 4.0 International License, which permits use, sharing, adaptation, distribution and reproduction in any medium or format, as long as you give appropriate credit to the original author(s) and the source, provide a link to the Creative Commons licence, and indicate if changes were made. The images or other third party material in this article are included in the article's Creative Commons licence, unless indicated otherwise in a credit line to the material. If material is not included in the article's Creative Commons licence and your intended use is not permitted by statutory regulation or exceeds the permitted use, you will need to obtain permission directly from the copyright holder. To view a copy of this licence, visit http://creativecommons.org/licenses/by/4.0/ The Creative Commons Public Domain Dedication waiver (http://creativecommons.org/publicdomain/zero/1.0/) applies to the data made available in this article, unless otherwise stated in a credit line to the data. 
phenotypic approach or a deficit accumulation approach $[5,6]$. In research, a commonly used approach to capture frailty is the Fried phenotype, which has been extensively tested for its validity $[7,8]$.

Frailty that combines a range of diverse deficits is increasingly recognized as a fundamental determinant of an individual's vulnerability or resilience to stressors [9] and has been linked to impaired cognition [10, 11]. Cognitive impairment has been shown to improve the predictive value of frailty, measured using the Fried phenotype, for adverse health outcomes [11]. Various neurocognitive disorders, including late-life cognitive impairment [12, 13], mild cognitive impairment (MCI) [14], dementia [15] and Alzheimer's disease (AD) $[16,17]$, have shown associations with frailty. Indeed, frailty moderates the association between AD pathology and the clinical expression of dementia, such that in the presence of frailty, even low AD pathological burden may manifest as dementia [17]. Researchers have also found that frailty and cognitive decline might share common physiological mechanisms, with greater frailty being associated with worse cognition and a faster rate of cognitive decline [18]. Thus, associations between frailty and other risk markers for cognitive decline are warranted.

Similar to frailty, neuropsychiatric symptoms (NPS) have demonstrated associations with cognitive decline and have been linked to known dementia biomarkers, thus also suggesting common underlying mechanisms. The Mayo Clinic Study of Aging reported that the presence of NPS (particularly agitation, apathy, anxiety, irritability or depression) was associated with an increased risk of developing MCI in cognitively normal older adults [19]. More recent evidence from a large sample in the National Alzheimer Coordinating Center dataset demonstrated that in $59 \%$ of dementia cases, NPS emerged in advance of cognitive symptoms, including $30 \%$ of people who developed $\mathrm{AD}$, reinforcing the notion that later-life onset of NPS can be an early marker of dementia [20]. To operationalize the assessment of NPS as risk markers for dementia, the International Society to Advance Alzheimer's Research and Treatment developed criteria for mild behavioral impairment (MBI) [21], which is a neurobehavioral syndrome characterized by later-life emergent NPS as an at-risk state for incident cognitive decline and dementia. Although MBI and MCI can co-occur, MBI can also precede $\mathrm{MCI}$, manifesting in older adults with subjective cognitive decline or even normal cognition, in whom MBI has demonstrated an increased risk of cognitive decline and dementia [22-26]. MBI may be the initial manifestation of neurodegeneration for some, and has been connected with known biomarkers for dementia including amyloid beta [27], tau [28, 29], neurofilament light [30], cortical atrophy [31, 32], white matter atrophy [33], and $\mathrm{AD}$ risk genes $[34,35]$. MBI has also been used in machine learning models to predict neurocognitive diagnostic category 40 months later [36]. These findings suggested that the early recognition of the NPS that constitute MBI may contribute to earlier detection of neurodegeneration, and may represent a clinical entity and premorbid treatment target to explore for intervention strategies to prevent or delay the onset of dementia [37]. The Mild behavioral impairment Checklist (MBI-C) is the validated brief screening instrument developed to capture MBI in accordance with the criteria [38-42].

Frailty, as a substantial moderator in the clinical expression of dementia, could be a predictor of cognitive decline over time [17, 43, 44]. However, the association between frailty and cognition in pre-dementia has yielded mixed results [45-47]. MBI is associated with a significantly faster rate of cognitive decline and progression along the continuum of neurodegenerative pathology compared to late life psychiatric disorders, and compared to those without MBI. Thus predictive value of MBI appears to be early in the neuropathological course of disease, in advance of cognitive impairment for some [22].

Identifying at-risk populations is an important public health issue, in order to explore risk reduction. The possible association between MBI and frailty, both independent risk factors for dementia appearing early in the disease course, should also be further investigated. In this cross-sectional study, we aimed to: 1) determine the prevalence of frailty and of MBI; 2) replicate prior findings linking frailty to worse objective global cognition; 3) determine the association between MBI and global cognition; and 4) assess the relationships between MBI total and domain scores, and frailty, in a primary care sample of older adults with at most mild cognitive impairment. We hypothesized that MBI would predict greater frailty burden.

\section{Methods \\ Participants and setting}

Altogether, a series of 185 volunteers aged 60 or older were consecutively recruited from the course of the Anti-Aging Study, aiming to investigates the association of frailty with health. All subjects were recruited through advertisements at the GPs clinics and Medical Management Centers in Guangzhou (the capital of the Guangdong, South-East of China). 1) aged 60 years or above; 2) ability to speak Chinese; 3) having adequate auditory and visual acuity; and 4) being able to provide wirtten informed consent to participate in the study. All participants were required to complete the eligibility assessment, including an elaborated medical record as well as neuropsychological assessment. Exclusion criteria included the following: 1) those with history of neurological and psychiatric illness,(eg, Alzheimer's disease, Parkinson's disease or dementia); 2) brain injury with 
loss of consciousness lasting at least $5 \mathrm{~min}$ or longer; and 3) any multisystemic or potentially life-threatening illness that might affect follow-up interviews. At enrollment, participants were asked to complete a comprehensive evaluation including but not limited to a structured questionnaire that collected demographic, medical record, medication review and clinical characteristics, and frailty assessment, emotional assessment and a neuropsychological assessment. Participants were excluded if they had a cognitive score consistent with dementia, defined as a Mini Mental State Examination (MMSE) cutoff score $\geq 24[48,49]$. Participants were also excluded if they were missing covariate data (Fig. 1 lists exclusion details).

\section{Sociodemographic and clinical characteristics}

In this study, we evaluated sociodemographic features including sex, age, and education. In addition, nutritional status was determined and classified according to the body mass index (BMI, $\left.\mathrm{kg} / \mathrm{m}^{2}\right)$, that was calculated as weight $(\mathrm{kg})$ divided by height in meters squared $\left(\mathrm{m}^{2}\right)$ [50]. To identify polypharmacy and multimorbidity, the subjects were asked whether they had a physician- determined diagnosis of hypertension, heart disease, diabetes mellitus, stroke, rheumatic disease, cancer, neurologic disease, osteoporosis (OP), lung disease, urinary incontinence or fecal incontinence. In general, polypharmacy has been defined as concurrent administration of more than 5 long-term medications, while multimorbidity as the coexistence of $>2$ chronic conditions [51].

\section{Emotional assessment}

In this study, Anxiety and depression were measured by the Generalized Anxiety Disorder 7-item (GAD-7) scale [52] and the 9-item Patient Health Questionnaire (PHQ9) [53], respectively. To be specific, the GAD-7 scale determines the frequency of the anxious mood items, which ranges from never (0) to almost every day (3), and the presence of an anxiety symptomatology is defined as a total score of $\geq 10$ [54]. On the other hand, the PHQ-9 scale determines the frequency of the depressed mood items, which ranges from not at all (0) to almost every day (3). The standard cut-off score of 10 or greater maximized the combined sensitivity and specificity in the primary studies [55]. As a result, the GAD-7 score of $\geq 10$ was utilized to be the threshold to indicate the

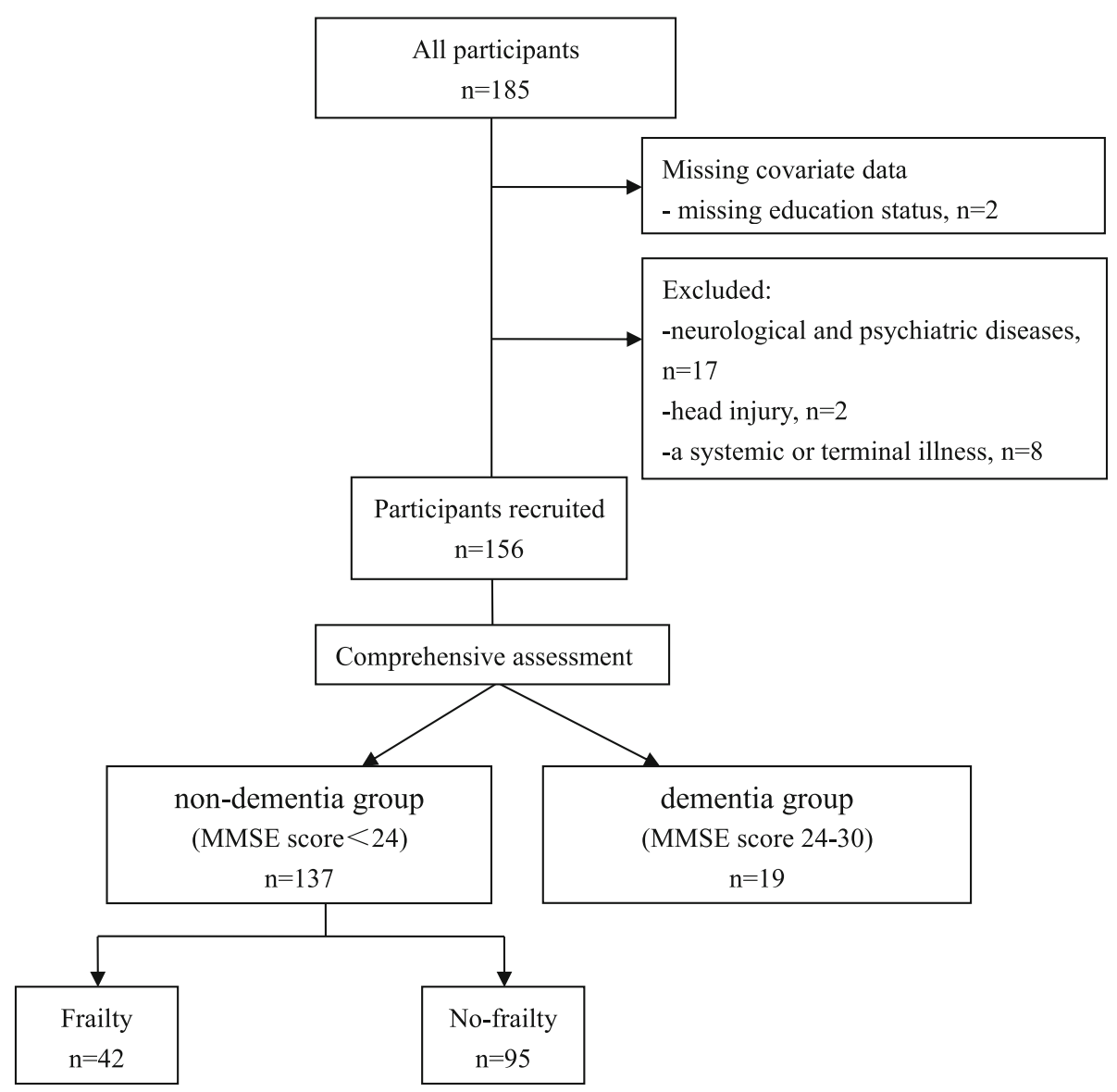

Fig. 1 Participant flow chart. Participant inclusion/exclusion criteria. Missing data categories are mutually exclusive 
clinically significant anxiety, and the PHQ-9 score $\geq 10$ indicates clinically significant depression, respectively [56].

\section{Frailty assessment}

The diagnosis of frailty was based on Fried phenotype in accordance with five indicators: exhaustion, which was based on a self-report questionnaire including two items extracted from the Center for Epidemiology Studies Depression (CES-D) [57]; unintentional weight loss (defined by the weight loss of $\geq 10$ pounds or $\geq 5 \%$ of body weight in last year); weak grip strength measured by the dominant grip-strength through the hand grip dynamometer and defined according to an established cutoffs by sex as well as body mass index (BMI); slowed gait speed (speed below an established cutoffs adjusted for sex and height), which was measured by walking time of $15 \mathrm{ft}$; and low energy expenditure (low level of physical activity over the last 2 weeks, after adjusting for sex), which was predicted by the Minnesota Leisure Time Physical Activity (MLTA) questionnaire [4]. Based on these scores, individuals with $0-2$ criteria present were categorized into the no-frailty group and those with 3 or more criteria were in the frailty group [58].

\section{Neuropsychiatric and neuropsychological assessment}

MBI was assessed using the Chinese version of MBI-C developed by Cui [59], a scale established specifically for the community elderly with independent function. The MBI-C [42] includes 34 items classified as five domains: 1) decreased motivation (apathy); 2) emotional dysregulation (symptoms of mood and anxiety disorders); 3) impulse dyscontrol (impulsivity, aggression and agitation); 4) social inappropriateness (impaired social cognition); and 5) abnormal perception or through content (psychotic symptoms, i.e. hallucination and delusions). Only symptoms that were characterized by emergence in later life, representing a change from longstanding patterns of behavior, along with symptoms lasting for at least 6 months, were evaluated as "yes", with the severity rated (1 to 3 points) [42]. The total scores of MBI-C domain were calculated by adding together the scores of five domains. MBI+ status was based on a total score $>8$, its optimal cut-off point for MBI case detection in a primary care population, with good sensitivity and specificity [39].

As part of the objective cognitive assessment, all subjects completed brief objective cognitive screening tools. The Chinese versions of the Montreal Cognitive Assessment (MoCA-BC, Chinese version) [60] together with the Mini-mental State Examination (MMSE, Chinese version) [61] were used to measure global cognitive function. In addition, The MoCA test includes more attention-executive items than the MMSE and was included to be sensitive for mid cognitive impairment, whereas the MMSE was used primarily to screen out dementia [62]. Both MMSE and MoCA were administered randomly for the sake of avoiding the fatigue effect bias. The potential scores range from 0 to 30 , with higher values indicating better cognition.

\section{Statistical analysis}

Continuous variables and categorical variables were presented as the mean \pm standard deviations (SD) and frequency/percentage respectively. Independent samples $\mathrm{t}$-tests were performed to determine the differences between the frailty and no-frailty groups, with respect to the continuous variables. Chi-square $\left(\chi^{2}\right)$ tests were used to identify the group differences for the categorical variables. As for the MBI-C scale, its total along with the domainspecific questionnaire scores were calculated. The distribution of the scores in MBI-C and the prevalence of MBI diagnosis were determined using frequency and descriptive analyses. In addittion, logistic regression analyse was performed to estimate odds ratios (ORs) with 95\% confidence intervals (95\% CIs) for the association between frailty status with age, education, depression, MBI and objective cognition. All analyses have been conducted using SPSS statistical analysis software, version 18.0. A twosided $P$-value of $<0.05$ was defined as the level of statistically significance throughout the analysis.

\section{Results}

\section{Participant characteristics}

There were 137 older adults enrolled in this study; the mean age was $69.6 \pm 7.6$ years, and the age range was 60 to 90 years old. Among these, 94 (68.6\%) were female, 21 $(15.3 \%)$ had a primary school education or lower, 43 (31.4\%) had multimorbidity, the presence of more than 2 comorbid conditions, and 27 (19.7\%) had polypharmacy (took five or more oral medications daily). Of these enrolled individuals, the mean BMI was $22.5 \pm 3.3 \mathrm{~kg} / \mathrm{m}^{2}$ and 31 (22.7\%) had depression symptoms, while 14 (10.4\%) had anxiety symptoms. According to the definition, 42 participants were categorized into the frailty group (30.7\%) and 95 into the no-frailty group (69.3\%). The frailty group showed worse performance on the MMSE (26.7 vs $28.0, p<0.05)$ and MoCA (25.2 vs 26.1 , $p<0.05)$ scores than the no-frailty group. The two groups also presented significant differences in age, education, comorbid conditions $>2$, polypharmacy and depression. No significant differences were found between the two groups with respect to sex, BMI and anxiety symptoms (Table 1 ).

A total of 25 (18.2\%) participants were MBI+, and 112 (81.8\%) were MBI-. Regarding group composition, the mean age of $\mathrm{MBI}+$ participants $(72.2 \pm 7.7)$ was higher than that of MBI- participants $(69.5 \pm 7.0)(p<0.05)$. The MBI+ individuals had significantly poorer cognition, 
Table 1 Characteristics of 137 Participants Aged $\geq 60$ Years and Stratified by MBI Status and Frailty Status

\begin{tabular}{|c|c|c|c|c|c|c|c|}
\hline \multirow[t]{2}{*}{ Variable } & \multirow{2}{*}{$\begin{array}{l}\text { Full } \\
\text { Sample } \\
(n= \\
137)\end{array}$} & \multicolumn{3}{|l|}{ MBI Status } & \multicolumn{3}{|l|}{ Frailty Status } \\
\hline & & $\mathrm{MBI}-(n=112)$ & $\mathrm{MBI}+(n=25)$ & $P$-value & No-frailty $(n=95)$ & Frailty $(n=42)$ & $P$-value \\
\hline Age, mean (SD) & $69.6(7.6)$ & $69.0(7.5)$ & $72.2(7.7)$ & .05 & $67.9(6.9)$ & $73.2(8.1)$ & $<.001$ \\
\hline Female & $94(68.6)$ & $86(76.8)$ & $18(72.0)$ & .61 & $58(61.1)$ & $36(85.7)$ & .07 \\
\hline Education & & & & .34 & & & .01 \\
\hline Primary or lower & $21(15.3)$ & $15(13.4)$ & $6(24.0)$ & & $10(10.5)$ & $11(26.2)$ & \\
\hline Completed high school & $75(54.7)$ & $64(57.1)$ & $11(44.0)$ & & $50(52.6)$ & $25(59.5)$ & \\
\hline At least some college & $41(29.9)$ & $33(29.5)$ & $8(32.0)$ & & $35(36.8)$ & $6(14.3)$ & \\
\hline Comorbid conditions $>2$ & $43(31.4)$ & $37(33.0)$ & $13(52.0)$ & .08 & $27(28.4)$ & $23(54.8)$ & .01 \\
\hline Polypharmacy & $27(19.7)$ & $22(19.6)$ & $5(20.0)$ & .97 & $9(9.5)$ & $18(42.9)$ & $<.001$ \\
\hline BMI, mean (SD) & $22.5(3.3)$ & $22.5(3.2)$ & $22.7(3.9)$ & .75 & $22.8(2.8)$ & $21.9(4.1)$ & .14 \\
\hline Depression (PHQ-9 $\geq 10$ ) & $31(22.7)$ & $26(23.2)$ & $5(20.0)$ & .73 & $16(16.8)$ & $15(35.7)$ & .02 \\
\hline Anxiety (GAD-7 $\geq 10$ ) & $14(10.2)$ & $12(10.7)$ & $2(8.0)$ & .69 & $8(8.4)$ & $6(14.3)$ & .30 \\
\hline MMSE, mean (SD) & $27.6(2.4)$ & $27.8(2.3)$ & $26.8(2.9)$ & .049 & $28.0(1.8)$ & $26.7(3.4)$ & .01 \\
\hline MoCA, mean (SD) & $25.8(2.5)$ & $26.1(2.2)$ & $24.7(3.2)$ & .009 & $26.1(2.2)$ & $25.2(2.9)$ & .04 \\
\hline
\end{tabular}

Notes: SD Standard deviation, MBI Mild behavioral impairment, MBI-C Mild Behavioral Impairment Checklist, BMI Body mass index; Results presented as $\mathrm{n}$ (\%) unless otherwise noted. Chi-square tests were used for categorical variables, whereas t-tests were used for continuous variables

with lower MMSE (26.8 vs 27.8, $p<0.05)$ and MoCA (24.7 vs $26.1, p<0.05$ ) scores than the MBI- individuals. No significant differences were found between the MBI+ individuals and MBI- individuals in terms of sex, education level, BMI, comorbid conditions $>2$, polypharmacy, or depression and anxiety symptoms $(p>0.05)$ (Table 1$)$.

\section{Frailty and mild behavioral impairment}

MBI status was significantly different between participants with and without frailty $(p=0.038)$. The MBI-C composite score was associated with frailty $(p=0.001)$. Of the five MBI domains, participants with decreased motivation, affective dysregulation and social inappropriateness MBI domains were more likely to have frailty. Neither impulse dyscontrol nor psychosis differed between frailty groups, although impulse dyscontrol neared statistical significance $(p=0.059)$ were found in our study (Table 2).

Multivariable logistic regression analysis indicated that MBI+ status was significantly associated with higher risk of having frailty, with an OR of 3.09 (95\% CI $=1.29$ 9.41; $p=0.047$ ) (Table 3). We also evaluated the associations between frailty status and global cognition, depression, education and age; we found that age and depression were significantly related to a higher risk of having frailty $(p<0.05)$, but the association with education, MMSE and MoCA score was not significant $(p>$ 0.05) (Table 3).

\section{Discussion}

To our knowledge, this is the first cross-sectional study to evaluate the relationships between frailty, MBI, and cognition. First, we determined that frailty is common in this population, with a prevalence of $30.7 \%$. Second, MBI was also fairly common, with a prevalence of $18.2 \%$. Third, greater burden of frailty was associated with poorer cognition, measured using the MMSE ( $p=$ $.01)$ and MoCA $(p=.04)$. Fourth, compared to those without MBI, MBI+ status was associated with poorer cognition measured using the MMSE $(p=.049)$ and

Table 2 Frailty and Cognitive and Behavioural Characteristics

\begin{tabular}{lllll}
\hline & Frailty $(\boldsymbol{n}=\mathbf{4 2})$ & No-frailty $(\boldsymbol{n}=\mathbf{9 5})$ & X2/F value & X2=4.3 \\
\hline MBI, $\mathrm{n}(\%)$ & $12(28.6)$ & $13(13.7)$ & $\mathrm{F}$ value \\
MBI score, mean (SD) & $7.3(5.2)$ & $4.7(3.6)$ & $\mathrm{F}=13.3$ & .038 \\
Decreased motivation & $2.2(2.2)$ & $1.3(1.2)$ & $\mathrm{F}=.6$ & .001 \\
Affective dysregulation & $1.8(1.4)$ & $1.3(1.1)$ & $\mathrm{F}=1.3$ & .005 \\
Impulse dyscontrol & $2.3(2.0)$ & $1.6(1.9)$ & $\mathrm{F}=12.5$ & .028 \\
Social inappropriateness & $0.7(1.0)$ & $0.4(.7)$ & $\mathrm{F}=5.0$ & .059 \\
Psychosis & $0.3(.7)$ & $0.2(.5)$ & & .246 \\
\hline
\end{tabular}

MBI Mild behavioral impairment, MBI-C Mild Behavioral Impairment Checklist 
Table 3 Multivariable logistic regression analysis for the association between frailty status and objective cognition with mild behavioral impairment

\begin{tabular}{llllll}
\hline & \multicolumn{6}{l}{ Frailty Status } \\
\cline { 2 - 6 } & $\boldsymbol{\beta}$ & Sr & Wal $\mathbf{2}$ & $\boldsymbol{\rho}$ value & Odds ratio $(\mathbf{9 5 \%} \mathbf{~ C l})$ \\
\hline Age & -.09 & .04 & 5.28 & .022 & $.91(.84-.99)$ \\
Education & -.69 & 1.10 & .40 & .529 & $.50(.06-4.3)$ \\
Depression & 1.62 & .51 & 10.27 & .001 & $5.04(1.88-13.58)$ \\
MoCA & -.13 & .15 & .73 & .392 & $.88(.66-1.18)$ \\
MMSE & .18 & .16 & 1.21 & .272 & $1.19(.87-1.64)$ \\
MBI & 1.13 & .57 & 3.95 & .047 & $3.09(1.29-9.41)$ \\
\hline
\end{tabular}

Abbreviations: Cl Confidence intervals

MoCA $(p=.01)$. Fifth, MBI+ status predicted higher levels of frailty $(\mathrm{OR}=3.09 ; 95 \% \mathrm{CI}=1.29-9.41)$, and this signal was driven by the MBI domains of decreased motivation, affective/emotional dysregulation, and social inappropriateness $(p<0.05)$. These results suggest that in non-demented older adults, frailty and MBI are both common and associated with small but significant impairment in global cognition.

The prevalence of frailty was $30.7 \%$ in our study, which was relatively high compared with previous estimates, which ranged from $11 \%$ up to $26 \%$ in community samples [63-65]. This difference may be attributed to our study design and to the fact that participants came from primary care clinics. Frailty may increase the risk of future cognitive decline, and that cognitive impairment may increase the risk of frailty, suggesting that cognition and frailty may interact in the cycle of agerelated decline $[66,67]$. Our results indicated that frailty was associated with age-related cognitive decline, describing an at-risk group for the preclinical phase of neurocognitive disorders, consistent with previous studies [11-16]. In their seminal study, Solfrizzi and colleagues reported that frail older adults had a higher prevalence of cognitive impairment than those without frailty (77\% vs. 54\%) [68]. Furthermore, components of frailty appeared to be related to pathological findings of $\mathrm{AD}$ and vascular dementia, supporting the idea of a possible common biological pathway between frailty and cognitive disorders [69]. A previous study found that there was an increase in neurons with cellular senescence and aging of microglia, and therefore, increases in apoptosis, aggregation of protein, and mitochondrial dysfunction, with increased reactive oxygen species, oxidative damage to proteins and lipids, and accumulation of DNA damage [69]. Accordingly, increasing frailty may be an indicator of future cognitive impairment.

The prevalence of MBI (18.2\%) in our participants was higher than that reported by Creese [22] in the PROTECT study, in which $10 \%$ of community-dwelling older adults aged 50 or over $(n=9931)$ reported MBI, as captured by the MBI-C. In a clinical sample of Spanish primary care patients from which the current cut-points were derived, the prevalence of MBI was $5.8 \%$ in older adults with subjective complaints [39] and 14.2\% in MCI [40]. These estimates collectively, determined using the $\mathrm{MBI}-\mathrm{C}$, are considerably lower than previous prevalence estimated generated using the Neuropsychiatric Inventory [70] which ranged from 28 to $51 \%$ in a community population [71, 72], and $49-85 \%$ in a cognitive neurology clinic population [71, 73]. These differences may be due to the diagnostic frame of reference of 1 month of symptoms captured by the Neuropsychiatric Inventory, whereas the MBI-C involves a more rigorous standard of six-month symptom duration and explicit laterlife onset of symptoms, in accordance with the MBI criteria. The lower MBI frequency generated using the $\mathrm{MBI}-\mathrm{C}$ reflects increased diagnostic specificity for $\mathrm{MBI}$, eliminating the inclusion of transient and reactive states, by excluding false positive symptoms.

Neuropsychiatric symptoms are associated with an increased risk of cognitive deficits across the lifespan, and MBI is associated with poorer cognition cross-sectionally [74], as well as longitudinally in comparison to those without MBI [23, 24]. In agreement with this previous evidence, we also found subtle but significant differences in global cognition reflected by lower scores on both the MMSE and $\mathrm{MoCA}$ in patients with MBI. Indeed, the MBI-C might have significantly higher discriminatory power than the MMSE when seeking to detect older adults with subtle cognitive decline [42]. Considering that MBI reflects the neurobehavioral axis of pre-dementia at-risk states and is a complement to the neurocognitive risk axis represented by MCI [31], this complementary approach may increase the yield when using both cognitive and behavioral approaches to screen for early-stage neurocognitive disorders.

In this study, we found that MBI was associated with higher levels of frailty, even after adjustment for potential confounders, and that this signal was driven by the MBI domains of decreased motivation, affective/emotional dysregulation and social inappropriateness. Our findings extend the literature by describing different patterns of association of MBI and its components with frailty, a pattern not previously established. Prior studies exploring the link between frailty and cognition have focused on individual functional abilities and assessed only global cognitive ability or limited cognitive domains $[14,75]$. The mechanisms for the association are not clear, but possibly involve abnormalities in biological processes related to aging [76]. A growing body of epidemiological evidence indicates that the mechanisms involved in the onset of frailty are also those that promote neurodegeneration, including chronic inflammation [66] and oxidative stress [77]. Other clinical polypharmacy and multimorbidity can increase the risk of both frailty and dementia $[78,79]$. 
MBI may serve as a proxy marker for frailty, or potentially a risk factor of frailty. Thus, MBI assessment may provide an approach to identify frailty early or to determine the risk of frailty in advance of completing a clinical assessment. This approach identifies potentially novel opportunities to prevent or delay frailty, age-related cognitive decline and other associated adverse health outcomes. The ease of administration of the MBI-C, which has been validated for telephone and online administration with high sensitivity and specificity $[38,39,74]$, positions it as a simple and cost-effective tool to be administered remotely or at scale for detecting those at clinical risk, in order to flag them for further assessment and work up.

The limitations of our study include the participant population and the sample size. Lower prevalence of MBI and frailty among participants in communities rather than clinical, hospital, or institutional settings are to be expected, and it is unclear if these results can be generalized. We had a limited sample size in this study, and replication with a larger sample is required. Hence, the clinical utility of the cognitive frailty construct cannot be unequivocally supported by this study, but it should be further investigated in future studies independently undertaken by other investigators in older populations. The frailty instrument may also present another limitation. Due to the constraints related to time, resources, and space, we chose Fried phenotype, combining five physical and physiological burden items, determined simply and quickly. Additional studies with other multidimensional and more elaborate objective assessments, representing as many domains as possible, are needed in order to validate these findings.

\section{Conclusion}

In conclusion, our findings provide further evidence that MBI and frailty are common among non-demented older adults, with both reflecting subtle but significant deficits in global cognition. MBI, especially in the domains of decreased motivation, affective dysregulation and social inappropriateness, is significantly associated with an increased risk of frailty in those with at most mild cognitive deficits. The MBI-C used in clinical practice could represent a simple and beneficial instrument for the detection of risk prior to the onset of frailty. Overall, these findings emphasize the importance of assessing physical as well as cognitive and behavioral function in older adults to identify risk. The inclusion of these measures in the assessment of frailty can improve the predictive validity of the phenotype regarding adverse health outcomes, and capture an at-risk group for early intervention.

\section{Abbreviations}

MBI: Mild behavioral impairment; NPS: Neuropsychiatric symptoms; MCl: Mild cognitive impairment; AD: Alzheimer's disease; NC: Normal cognition; MBIC: Mild Behavioral Impairment Checklist; FP: The Fried phenotype; MoCA-
BC: Chinese versions of the Montreal Cognitive Assessment; MMSE: Minimental State Examination; BMI: Body mass index; ANOVA: Analysis of variance; ORs: Odds ratios; Cls: Confidence intervals

\section{Acknowledgments \\ We appreciate the cooperation of the staff of the Guangdong Provincial Hospital of Chinese Medicine.}

\section{Authors' contributions}

SF, ZY and FX conceived and designed the study. SF, XL and TY recruited the participants, collected the data for the manuscript and provided substantial feedback. SF, ZP, ZI and BH analyzed and interpreted the data. SF, $\mathrm{ZI}, \mathrm{ZY}$ and FX wrote the first draft of the manuscript. All authors read and approved the final manuscript.

\section{Funding \\ The study was supported by the National Key R\&D Program of China, No. 2018YFC1705600; Guangdong Provincial Hospital of Chinese Medicine's Scientific and Technological Plan, No. YN2016ZWB07 and Guangdong Research Center for TCM Services and Industrial Development, No.2019YBB06. Funders played no role in this study (the design, data collection, execution, analysis, interpretation of data and writing the manuscript).}

\section{Availability of data and materials}

The datasets used and/or analyzed during the current study are available from the corresponding author on reasonable request.

\section{Ethics approval and consent to participate}

This study was approved by the Ethics Review Board of Guangdong Provincial Hospital of Chinese Medicine Ethics Committee (reference: B2017168-01) and all the participants provided written informed consent. For some participants recognized as having cognitive impairment and/or severe illness, we obtained proxy consent from a family member or another supportive adult on their behalf.

\section{Consent for publication}

Not applicable.

\section{Competing interests}

The authors declare that they have no competing interests.

\section{Author details}

${ }^{1}$ The Second Clinical College of Guangzhou University of Chinese Medicine, 232 East Ring Road, Guangzhou, P. R. China. ${ }^{2}$ Department of Neurology, First Affiliated Hospital of Sun Yat-sen University, 58 Zhongshan Second Road, Guangzhou, P. R. China. ${ }^{3}$ Division of Translational Neuroscience, Department of Clinical Neurosciences, Hotchkiss Brain Institute, Alberta Children's Hospital Research Institute, Cumming School of Medicine, University of Calgary, 3330 Hospital Drive NW, Calgary, AB T2N 4N1, Canada. ${ }^{4}$ Departments of Psychiatry, Clinical Neurosciences, and Community Health Sciences, Hotchkiss Brain Institute and O'Brien Institute for Public Health, University of Calgary, Calgary, Canada. ${ }^{5}$ The Second Affiliated Hospital of Guangzhou University of Chinese Medicine, Guangdong Provincial Hospital of Chinese Medicine, 111 Dade Road, Guangzhou, People's Republic of China.

Received: 10 June 2020 Accepted: 12 November 2020

Published online: 27 November 2020

\section{References}

1. Morley JE, Vellas B, van Kan GA, Anker SD, Bauer JM, Bernabei R, et al. Frailty consensus: a call to action. J Am Med Dir Assoc. 2013;14(6):392-7.

2. Song $X$, Mitnitski A, Rockwood K. Prevalence and 10-year outcomes of frailty in older adults in relation to deficit accumulation. J Am Geriatr Soc. 2010; 58(4):681-7

3. Siriwardhana DD, Hardoon S, Rait G, Weerasinghe MC, Walters KR. Prevalence of frailty and prefrailty among community-dwelling older adults in low-income and middle-income countries: a systematic review and metaanalysis. BMJ Open. 2018;8(3):e018195. 
4. Fried LP, Tangen CM, Walston J, Newman AB, Hirsch C, Gottdiener J, et al. Frailty in older adults evidence for a phenotype. J Gerontol. 2001;56(3): M146.

5. Searle SD, Mitnitski A, Gahbauer EA, Gill TM, Rockwood K. A standard procedure for creating a frailty index. BMC Geriatr. 2008;8:24.

6. Rockwood K, Andrew M, Mitnitski A. A comparison of two approaches to measuring frailty in elderly people. J Gerontol A Biol Sci Med Sci. 2007;62(7): 738-43.

7. Pritchard JM, Kennedy CC, Karampatos S, loannidis G, Misiaszek B, Marr S, et al. Measuring frailty in clinical practice: a comparison of physical frailty assessment methods in a geriatric out-patient clinic. BMC Geriatr. 2017;17(1):264

8. Buta BJ, Walston JD, Godino JG, Park M, Kalyani RR, Xue QL, et al. Frailty assessment instruments: systematic characterization of the uses and contexts of highly-cited instruments. Ageing Res Rev. 2016;26:53-61

9. Canevelli M, Troili F, Bruno G. Reasoning about frailty in neurology: neurobiological correlates and clinical perspectives. J Frailty Aging. 2014; 3(1):18-20.

10. Panza F, Lozupone M, Solfrizzi V, Sardone R, Dibello V, Di Lena L, et al. Different cognitive frailty models and health- and cognitive-related outcomes in older age: from epidemiology to prevention. J Alzheimers Dis. 2018;62(3):993-1012

11. Avila-Funes JA, Amieva H, Barberger-Gateau P, Le Goff M, Raoux N, Ritchie K, et al. Cognitive impairment improves the predictive validity of the phenotype of frailty for adverse health outcomes: the three-city study. J Am Geriatr Soc. 2009;57(3):453-61.

12. Sugimoto T, Sakurai T, Ono R, Kimura A, Saji N, Niida S, et al. Epidemiological and clinical significance of cognitive frailty: a mini review. Ageing Res Rev. 2018;44:1-7.

13. Dartigues JF, Amieva H. Cognitive frailty: Rational and definition from an (I.a.N a./i.a.g.g.) international consensus group. J Nutr Health Aging. 2014;18(1):95.

14. Thibeau S, McDermott K, McFall GP, Rockwood K, Dixon RA. Frailty effects on non-demented cognitive trajectories are moderated by sex and Alzheimer's genetic risk. Alzheimers Res Ther. 2019;11(1):55.

15. Rogers NT, Steptoe A, Cadar D. Frailty is an independent predictor of incident dementia: evidence from the English longitudinal study of ageing. Sci Rep. 2017;7(1):15746.

16. Francesco P, Vincenzo S, Davide S, Imbimbo BP, Rosa C, Nicola Q, et al. Agerelated hearing impairment and frailty in Alzheimer's disease: interconnected associations and mechanisms. Front Aging Neurosci. 2015;7:113.

17. Wallace LM, Theou O, Godin J, Andrew MK, Bennett DA, Rockwood K. Investigation of frailty as a moderator of the relationship between neuropathology and dementia in Alzheimer's disease: a cross-sectional analysis of data from the rush memory and aging project. Lancet Neurol. 2019:18(2):177-84.

18. Fougère B, Delrieu J, Campo ND, Soriano G, Vellas B. Cognitive frailty : mechanisms, tools to measure, prevention and controversy. Clin Geriatr Med. 2017:33:339-55.

19. Geda YE, Roberts RO, Mielke MM, Knopman DS, Rocca WA. Baseline Neuropsychiatric Symptoms and the Risk of Incident Mild Cognitive Impairment: A Population-Based Study. Am J Psychiatry. 2014;7(4):S692.

20. Wise EA, Rosenberg PB, Lyketsos CG, Leoutsakos J-M. Time course of neuropsychiatric symptoms and cognitive diagnosis in National Alzheimer's coordinating centers volunteers. Alzheimer's \& Dementia: Diagnosis, Assessment \& Disease Monitoring. 2019;11:333-9.

21. Ismail Z, Smith EE, Geda Y, Sultzer D, Brodaty H, Smith G, et al. Neuropsychiatric symptoms as early manifestations of emergent dementia: provisional diagnostic criteria for mild behavioral impairment. Alzheimers Dement. 2016;12(2):195-202.

22. Creese B, Brooker H, Ismail Z, Wesnes KA, Hampshire A, Khan Z, et al. Mild behavioral impairment as a marker of cognitive decline in cognitively Normal older adults. Am J Geriatr Psychiatry. 2019;27(8):823-34.

23. Matsuoka T, Ismail Z, Narumoto J. Prevalence of mild behavioral impairment and risk of dementia in a psychiatric outpatient clinic. J Alzheimers Dis. 2019;70(2):505-13.

24. Ismail Z, McGirr A, Gill S, Hu S, Forkert ND, Smith EE. Mild Behavioral Impairment and Subjective Cognitive Decline predict Mild Cognitive Impairment. medRxiv. 2020; 2020.05.24.20112284..

25. Nathan S, Gill S, Ismail Z. APOE $\varepsilon 4$ status in pre-dementia risk states, mild behavioural impairment and subjective cognitive decline, and the risk of incident cognitive decline. 2020 Alzheimer's Association International Conference; 2020.
26. Taragano FE, Allegri RF, Heisecke SL, Martelli MI, Feldman ML, Sánchez $V$, et al. Risk of conversion to dementia in a mild behavioral impairment group compared to a psychiatric group and to a mild cognitive impairment group. J Alzheimers Dis. 2018;62(1): 227-38.

27. Lussier FZ, Pascoal TA, Chamoun M, Therriault J, Tissot C, Savard M, et al. Mild behavioral impairment is associated with $\beta$-amyloid but not tau or neurodegeneration in cognitively intact elderly individuals. Alzheimers Dement. 2020;16:192-9.

28. Johansson M, Smith R, Stomrud E, Johansson P, Janelidze S, van Westen D, et al. Mild behavioral impairment is predictive of tau deposition in the earliest stages of Alzheimer's disease. 2020 Alzheimer's Association International Conference; 2020.

29. Lussier FZ, Pascoal TA, Chamoun M, Therriault J, Tissot C, Savard M, et al. Mild behavioral impairment is associated with $\beta$-amyloid but not tau or neurodegeneration in cognitively intact elderly individuals. Alzheimers Dement. 2020;16(1):192-9.

30. Naude J, Gill S, Hu S, McGirr A, Forkert N, Monchi O, et al. Plasma Neurofilament light: a marker of cognitive decline in mild Behavioural impairment. J Alzheimers Dis. 2020;76(3):1017-27.

31. Yoon E, Ismail Z, Hanganu A, Kibreab M, Hammer T, Cheetham J, et al. Mild behavioral impairment is linked to worse cognition and brain atrophy in Parkinson's disease. Neurology. 2019;93(8):e766-e77.

32. Matuskova V, Ismail Z, Nikolai T, Markova H, Cechova K, Laczó J, et al. Mild behavioral impairment is associated with atrophy in Alzheimer's diseaserelated regions in non-demented older adults. 2020 Alzheimer's Association International Conference; 2020.

33. Gill S, Wang M, Forkert ND, MacMaster FP, Smith EE, Ismail Z. Diffusion Tensor Imaging in pre-dementia risk states: white matter atrophy findings in Mild Behavioral Impairment (P5. 1-025). Neurology. 2019;92(15 Supplement P5):1-025.

34. Andrews SJ, Ismail Z, Anstey KJ, Mortby M. Association of Alzheimer's genetic loci with mild behavioral impairment. Am J Med Genet B Neuropsychiatr Genet. 2018;177(8):727-35.

35. Creese B, Brooker H, Aarsland D, Corbett A, Ballard C, Ismail Z. Genetic risk for Alzheimer disease, cognition and Mild Behavioral Impairment in healthy older adults. medRxiv. 2020; 2020.05.13.20100800.

36. Gill S, Mouches P, Hu S, Rajashekar D, MacMaster FP, Smith EE, et al. Using machine learning to predict dementia from neuropsychiatric symptom and neuroimaging data. J Alzheimers Dis. 2020;75(1):277-88.

37. Gosselin PA, Ismail Z, Faris PD, Benkoczi CL, Fraser TL, Cherry SW, et al. Effect of hearing ability and mild Behavioural impairment on MoCA and memory index scores. Can Geriatr J. 2019;22(3):165.

38. Creese B, Griffiths A, Brooker H, Corbett A, Aarsland D, Ballard C, et al. Profile of mild behavioral impairment and factor structure of the mild behavioral impairment checklist in cognitively Normal older adults. Int Psychogeriatr. 2020;32(6):705-17.

39. Mallo SC, Ismail Z, Pereiro AX, Facal D, Lojo-Seoane C, Campos-Magdaleno $M$, et al. Assessing mild behavioral impairment with the mild behavioral impairment checklist in people with subjective cognitive decline. Int Psychogeriatr. 2019;31(2):231-9.

40. Mallo SC, Ismail Z, Pereiro AX, Facal D, Lojo-Seoane C, Campos-Magdaleno $M$, et al. Assessing mild behavioral impairment with the mild behavioral impairment-checklist in people with mild cognitive impairment. J Alzheimers Dis. 2018;66(1):83-95.

41. Hu S, Patten SB, Fick G, Smith EE, Ismail Z. Validation of the mild behavioral impairment checklist (MBI-C) in a clinic-based sample. Alzheimer's Dementia. 2019;15(7):365.

42. Ismail Z, Aguera-Ortiz L, Brodaty H, Cieslak A, Cummings J, Fischer CE, et al. The mild behavioral impairment checklist (MBI-C): a rating scale for neuropsychiatric symptoms in pre- dementia populations. J Alzheimers Dis. 2017:56(3):929-38.

43. Song $X$, Mitnitski A, Rockwood K. Nontraditional risk factors combine to predict Alzheimer disease and dementia. Neurology. 2011;77(3):227-34

44. Kojima G, Taniguchi Y, lliffe S, Walters K. Frailty as a predictor of Alzheimer disease, vascular dementia, and all dementia among community-dwelling older people: a systematic review and meta-analysis. J Am Med Dir Assoc. 2016:17(10):881-8

45. Boyle PA, Buchman AS, Wilson RS, Leurgans SE, Bennett DA. Physical frailty is associated with incident mild cognitive impairment in community-based older persons. J Am Geriatr Soc. 2010;58(2):248-55. 
46. Robertson DA, Savva GM, Coen RF, Kenny R-A. Cognitive function in the Prefrailty and frailty syndrome. J Am Geriatr Soc. 2014;62(11):2118.

47. Chen S, Honda T, Narazaki K, Chen T, Nofuji Y, Kumagai S. Global cognitive performance and frailty in non-demented community-dwelling older adults: findings from the Sasaguri Genkimon study. Geriatr Gerontol Int. 2016;16(6): 729-36.

48. Terazawa S, Oshima H, Narita Y, Fujimoto K, Mutsuga M, Tokuda Y, et al. Strategy of cardiovascular surgery for patients with dementia as evaluated by mini-mental state examination. Circ J. 2018;82(12):2998-3004.

49. Donohue MC, Sperling RA, Petersen R, Sun CK, Weiner MW, Aisen PS. Association between elevated brain amyloid and subsequent cognitive decline among cognitively normal persons. JAMA. 2017;317(22):2305-16.

50. Lipschitz DA. Screening for nutritional status in the older. Prim Care. 1994; 21(1):55-67.

51. Stawicki SP, Kalra S, Jones C, Justiniano CF, Papadimos TJ, Galwankar SC, et al. Comorbidity polypharmacy score and its clinical utility: a pragmatic practitioner's perspective. J Emerg Trauma Shock. 2015;8(4):224.

52. Spitzer RL, Kroenke K, Williams JBW, Löwe B. A brief measure for assessing generalized anxiety disorder: the GAD-7. Arch Intern Med. 2006;166(10): 1092-7.

53. Kroenke K, Spitzer RL, Williams JBW. The PHQ-9 : Validity of a Brief Depression Severity Measure. J Gen Intern Med. 2001;16(9):606-13.

54. Lowe B, Decker O, Muller S, Brahler E, Schellberg D, Herzog W, et al. Validation and standardization of the generalized anxiety disorder screener (GAD-7) in the general population. Med Care. 2008:46(3):266-74.

55. Levis B, Benedetti A, Thombs BD. Accuracy of Patient Health Questionnaire9 (PHQ-9) for screening to detect major depression: individual participant data meta-analysis. BMJ (Clinical research ed). 2019;365:11476.

56. Witthöft M, Hiller W, Loch N, Jasper F. The latent structure of medically unexplained symptoms and its relation to functional somatic syndromes. Int J Behav Med. 2013;20(2):172-83.

57. Orme JG, Reis J, Herz EJ. Factorial and discriminant validity of the Center for Epidemiological Studies Depression (CES-D) scale. J Clin Psychol. 1986;42(1):28-33.

58. Kojima G, Taniguchi $Y$, lliffe S, Urano T, Walters K. Factors associated with improvement in frailty status defined using the frailty phenotype: a systematic review and meta-analysis. J Am Med Dir Assoc. 2019;20(12): 1647-1649.e2

59. Cui Y, Dai S, Miao Z, Zhong Y, Liu Y, Liu L, et al. Reliability and validity of the Chinese version of the mild behavioral impairment checklist for screening for Alzheimer's disease. J Alzheimer's Dis. 2019:70(3):747-56.

60. Chen K-L, Xu Y, Chu A-Q, Ding D, Liang X-N, Nasreddine ZS, et al. Validation of the Chinese version of Montreal cognitive assessment basic for screening mild cognitive impairment. J Am Geriatr Soc. 2016;64(12):e285.

61. Richardson C, Stephan BCM, Robinson L, Brayne C, Matthews FE. Twodecade change in prevalence of cognitive impairment in the UK. Eur $J$ Epidemiol. 2019;34(11):1085-92.

62. Koski L. Validity and applications of the Montreal cognitive assessment for the assessment of vascular cognitive impairment. Cerebrovascular Dis. 2013; 36(1):6-18.

63. Li CL, Chang HY, Stanaway FF. Combined effects of frailty status and cognitive impairment on health-related quality of life among community dwelling older adults. Arch Gerontol Geriatr. 2020;87:103999.

64. Arnadottir SA, Bruce J, Lall R, Withers EJ, Underwood M, Shaw F, et al. The importance of different frailty domains in a population based sample in England. BMC Geriatr. 2020;20(1):16

65. Teo N, Yeo PS, Gao Q, Nyunt MSZ, Foo JJ, Wee SL, et al. A bio-psycho-social approach for frailty amongst Singaporean Chinese community-dwelling older adults - evidence from the Singapore longitudinal aging study. BMC Geriatr. 2019:19(1):350.

66. Panza F, Solfrizzi V, Frisardi V, Maggi S, Sancarlo D, Adante F, et al. Different models of frailty in predementia and dementia syndromes. J Nutr Health Aging. 2011;15(8):711-9.

67. Robertson DA, Savva GM, Kenny RA. Frailty and cognitive impairment-a review of the evidence and causal mechanisms. Ageing Res Rev. 2013;12(4):840-51.

68. Solfrizzi V, Scafato E, Seripa D, Lozupone M, Imbimbo BP, D'Amato A, et al. Reversible Cognitive Frailty, Dementia, and All-Cause Mortality. The Italian Longitudinal Study on Aging. J Am Med Dir Assoc. 2017;18(1):89.e1-8.

69. Aguilar-Navarro SG, Mimenza-Alvarado AJ, Anaya-Escamilla A, GutierrezRobledo LM. Frailty and Vascular Cognitive Impairment: Mechanisms Behind the Link. Rev Invest Clin. 2016:68(1):25-32.
70. Kaufer DI, Cummings JL, Ketchel P, Smith V, MacMillan A, Shelley T, et al Validation of the NPI-Q, a brief clinical form of the neuropsychiatric inventory. J Neuropsychiatry Clin Neurosci. 2000;12(2):233-9.

71. Mortby ME, Ismail Z, Anstey KJ. Prevalence estimates of mild behavioral impairment in a population-based sample of pre-dementia states and cognitively healthy older adults. Int Psychogeriatr. 2018;30(2):221-32.

72. Geda YE, Roberts RO, Knopman DS, Petersen RC, Christianson TJ, Pankratz VS, et al. Prevalence of neuropsychiatric symptoms in mild cognitive impairment and normal cognitive aging: population-based study. Arch Gen Psychiatry. 2008;65(10):1193-8.

73. Sheikh F, Ismail Z, Mortby ME, Barber P, Cieslak A, Fischer K, et al. Prevalence of mild behavioral impairment in mild cognitive impairment and subjective cognitive decline, and its association with caregiver burden. Int Psychogeriatr. 2018;30(2):233-44.

74. Kassam F, Chen H-Y, Nosheny RL, Williams T, Mackin RS, Weiner MW, et al. Cognitive profile of mild behavioral impairment (MBI) in Brain Health Registry participants. 2020 Alzheimer's Association International Conference; 2020

75. Brigola AG, Ottaviani AC, Carvalho DHT, Oliveira NA, Souza EN, Pavarini SCI. Association between cognitive impairment and criteria for frailty syndrome among older adults. Arq Neuropsiquiatr. 2020;78(1):2-8.

76. Fabricio DM, Chagas MHN, Diniz BS. Frailty and cognitive decline. Transl Res. 2020;221:58-64.

77. Mulero J, Zafrilla P, Martinez-Cacha A. Oxidative stress, frailty and cognitive decline. J Nutr Health Aging. 2011;15(9):756-60.

78. Moon JH, Huh JS, Won CW, Kim HJ. Is Polypharmacy associated with cognitive frailty in the elderly? Results from the Korean frailty and aging cohort study. J Nutr Health Aging. 2019;23(10):958-65.

79. Afilalo J, Karunananthan S, Eisenberg MJ, Alexander KP, Bergman H. Role of frailty in patients with cardiovascular disease. Am J Cardiol. 2009;103(11): 1616-21.

\section{Publisher's Note}

Springer Nature remains neutral with regard to jurisdictional claims in published maps and institutional affiliations.
Ready to submit your research? Choose BMC and benefit from:

- fast, convenient online submission

- thorough peer review by experienced researchers in your field

- rapid publication on acceptance

- support for research data, including large and complex data types

- gold Open Access which fosters wider collaboration and increased citations

- maximum visibility for your research: over $100 \mathrm{M}$ website views per year

At $\mathrm{BMC}$, research is always in progress.

Learn more biomedcentral.com/submission 